\title{
Cronobacter sakazakii: An Emerging Foodborne Bacterial Pathogen of Public Health Significance
}

\section{Mahendra Pal ${ }^{1 *}$, Yodit Ayale ${ }^{2}$ and Judit Molnar ${ }^{3}$}

${ }^{1}$ Narayan Consultancy on Veterinary Public Health and Microbiology, Aangan, Gujarat, India

${ }^{2}$ College of Agriculture and Natural Resources, Department of Animal Science, Bonga, Ethiopia

${ }^{3}$ Subregional Unified Social Institution, 9200 Mosonmagyaróvár Palánk u, Hungary

*Corresponding Author: Founder of Narayan Consultancy on Veterinary Public Health and Microbiology, Aangan, Gujarat, India.

Received: October 15, 2019; Published: October 24, 2019

DOI: $10.31080 /$ ASMI.2019.02.0410

\begin{abstract}
Foodborne pathogens have a great impact on health and economy of the country. Cronobacter sakazakii is an important foodborne pathogen associated with outbreaks of life-threatening necrotizing enterocolitis, meningitis, and sepsis in neonates and infants. It is an emerging bacterial foodborne pathogen that is reported from many countries of the world including India. There has been increasing interest among the whole public community and food industry, especially in the production of powdered infant formula. Hitherto, the natural habitat of $C$. sakazakii is unidentified. Clinical manifestations of disease include septicemia, meningitis and necrotizing enterocolitis. The contaminated powdered infant formula serves as an important vehicle of the bacteria. Cronobacter sakazakii has been isolated from various food products, such as mixed salad vegetables, meat, milk, and cheese. The organism is widely prevalent in the environment, plant materials, powdered infant formulas, cereal foods, fermented beverages, fruits, and vegetables. Contamination is caused by poor handling and added raw ingredients. As infants and young children are more vulnerable to foodborne infections, the microbiological safety of infants and powdered infant formula is more important. Cronobacter sakazakii can be controlled during the initial production of powdered infant formula, and avoiding post processing contamination, using suitable microbiological approaches. Further, regulatory standards for infant food manufacturers need to be improved.
\end{abstract}

Keywords: Cronobacter sakazakii; Emerging Pathogen; Foods; Infants; Life Threatening Infection; Mortality

\section{Introduction}

Foodborne diseases have emerged as an important growing public health and economic problem in many countries of the world during the last two decades. It comprises a broad group of illnesses concerning to public health problems. Among them, gastroenteritis is the most frequent clinical syndrome, which can be attributed to a wide range of microorganisms, including bacteria, viruses, and parasites [1]. Currently, over 250 foodborne diseases of varied etiologies have been described [2]. In USA, five pathogens, namely Staphylococcus aureus, Clostridium perfringenes, Salmonells, Campylobacter, and Norovirus are most commonly implicated in foodborne diseases. Further, infections caused by Listeria monocytogenes, Clostridium botulinum, Escherichia coli, and Vibrio is more likely to lead to hospitalization of the patient [2]. Recent decades have witnessed the emergence of many foodborne bacteria, which include Aeromonas hydrophila, Arcobacter butzlari, Campylobacter jejuni, Clostridium difficile, Cronobacter sakazakii, Escherichia coli 0157: H7, Listeria monocytogenese, Plesiomonas shigelloides, Vibrio parahaemolyticus, and Yersinia enterocolitica [3]. There seems to paucity of statistical data on the exact incidence and prevalence of foodborne diseases in most of the countries of world. In this context, CDC [2] estimated that foodborne illnesses are responsible for 48 million cases, 128,000 hospitalization and 3,000 deaths annually in the United States. 
Cronobacter species are emerging and opportunistic foodborne pathogens, which cause infections in neonates, infants, adults and immunocompressed individuals [4]. The primary reservoirs of Cronobacter are not well defined due to the ubiquitous nature of this bacterium. Cronobacter strains have been isolated from a wide range of foods including milk, cheese, dried foods, meats, water, vegetables, rice, tea, herbs, and spices, besides various food production environments [5].

Currently, seven are main internationally recognized species of Cronobacter, namely, C. sakazakii, C. turicensis, C. malonaticus, $C$. muytjensii, $C$. universalis, $C$. dublinensis and $C$. condiment [6]. Three of these namely, C. sakazakii, C. malonaticus, and C. turicensis, are human opportunistic pathogens [7] and the rest of them have not been associated with human disease until recently [8].

Cronobacter sakazakii, previously known as Enterobacter sakazakii, is an important foodborne pathogen, which is implicated with outbreaks of life-threatening necrotizing enterocolitis, meningitis, and sepsis in neonates and infants [9]. Bowen and Braden [10] mentioned that case definition for invasive E. sakazakii infections in infants have been met in only 46 cases worldwide. The infection caused by $C$. sakazakii can occur in sporadic and epidemic form. Ray and co-investigators [11] are credited to delineate the first report of E. sakazakii infections in infants from India. It is an emerging foodborne pathogen that causes life-threatening meningitis [12], septicemia, and enterocolitis in infants [13].

It is a Gram-negative, oxidase positive, catalase positive, nonspore-forming bacterium that belongs to the Enterobacteriaceae family $[14,15]$. This pathogen is receiving increasingly raised interest among the whole public community and food industry, especially in the production of powdered infant formula. The natural habitat of $C$. sakazakii is presently unknown. However, the organism has been recovered from water, sediment and soil. In a large survey of over 500 foodstuffs and ingredients, a large proportion $(\sim 25 \%)$ of herbs and spices were shown to contain C. sakazakii [14]. It is pertinent to mention that E. sakazakii has been isolated from milk powder manufacturing facilities and household vacuum cleaners [16] and also from baby foods, milk powders, cheese products, sausage meat, minced beef, and vegetables [14]. A recent study undertaken by Mahindroo and co-workers [17] indicated that the incidence of $C$. sakazakii in humans, goat meat, goat feces, pork, pig stool, chicken stool, and environment was 3.37\%,
$9.4 \%, 11.1 \%, 5.9 \%, 7.0 \%, 6.7 \%$, and $11.5 \%$, respectively. Antibiotic susceptibility profile revealed that of the 63 isolates of $C$. sakazakii tested, maximum resistance was noticed against ampicillin (50.8\%) followed by ciprofloxacin (20.6\%), and meropenem (17.2\%) [17].

Symptoms of Cronobacter sakazakii infection are severe, including meningitis, septicemia and necrotizing enterocolitis [18]. The original reservoir of $C$. sakazakii is still not known [19]. The bacterium has been isolated from various food products, such as mixed salad vegetables, milk, cheese, and meat [20]. The present communication delineates the growing significance of Cronobacter sakazakii as an emerging foodborne pathogen of public health concern.

\section{Clinical spectrum}

Neonates having $<2.5 \mathrm{~kg}$ weight and infants of $<28$ days age are at heightened risk compared to more mature infants [18]. Symptoms include meningitis leading to ventriculitis, brain abscess, hydrocephalus and cyst formation as well as necrotizing enterocolitis characterized by intestinal necrosis and pneumatosis intestinalis, pulmonary, urinary, and blood stream infections [21]. Infants that survive $C$. sakazakii infection often suffer delayed neurological symptoms, e.g., delayed brain development, brain abscesses or hydrocephalus [22]. The mortality rate for neonatal infections has been reported to be as high as $80 \%$ [23] and survivors often suffer from severe irreversible neurological disorders. Food other than infant formula has been rarely investigated for the presence of $C$. sakazakii. Nevertheless, this microorganism could be isolated from a wide spectrum of food and food ingredients. The International Commission on Microbiological Specification for Foods has classified C. sakazakii as a severe hazard for restricted populations, life-threatening or with substantial chronic sequelae over long duration [24].

\section{Transmission}

The sources of $C$. sakazakii and its vehicles of transmission are not clearly documented. It is distributed and frequently contaminated in the environment [25], plant materials [26], powdered infant formulas [27], cereal foods [28], fermented beverages [29], fruits, and vegetables [26]. In particular, contamination on powdered infant formula occurs more easily because it is a non-sterilized product. Contamination can be caused by poor handling and added raw ingredients. The information on environmental source, transmission routes, and human carriage of $C$. sakazakii in India is grossly lacking [17]. 


\section{Public health implications}

Infants and young children are particularly vulnerable to food borne infections. As result, the microbiological safety of infant and follow-up formula is very essential [30]. Because powered infant formula (PIF) is not a sterile product, it is an excellent medium to support bacterial growth. As we know bovine milk is a vital ingredient of PIF and a potential source of bacteria that are pathogenic to humans [31]. The World Health Organization recommends that infants should be exclusively breast-fed for the first 6 months of life. Infants who are not breast-fed should be provided with a suitable breast milk substitute, formulated in accordance with Codex Alimentarius Commission standards. To reduce the risk of infection in infants fed PIF, recommendations have been made for the preparation and storage of PIF. Manufacturers of PIF is being encouraged to develop a greater range of commercially sterile alternative formula products for high-risk groups. In addition, formula manufacturers must implement strategies aimed at reducing the risks of product contamination [32].

Prevention and control

Cronobacter sakazakii can be controlled during the initial production of PIF and avoiding post processing contamination, using suitable microbiological approaches, will have a positive effect. Standardized analytical approaches are necessary to ensure the safety of the product [33].

Effective environmental monitoring programs, good manufacturing practice guidelines, and hazard analysis and critical control point systems to control the risk of microbiological contamination along the entire production chain, from the starting raw materials, throughout the entire process, until the final product is ready for distribution, should be implemented [34]. In addition, health education should be imparted to the mothers to give breast feeding to child for at least 6 months.

\section{Conclusion}

Cronobacter sakazakii is an emerging foodborne bacterium that can cause life threatening infections particularly in neonates and infants. The infection can occur in sporadic as well as in epidemic form. The fatality rate in neonates may reach to $80 \%$. The infection caused by $C$. sakazakii is not well reported in many countries of the world including India. The pathogen has been isolated from a wide range of foods including, milk, meat, cereals and vegetables. Laboratory help is mandatory to make an unequivocal diagnosis of $C$. sakazakii infection. Cronobacter sakazakii can be controlled during the initial production of PIF and avoiding post processing contamination, by employing suitable microbiological approaches. It is emphasized that regulatory standards for infant food manufacturers need to be enhanced. Comprehensive and systematic research is required to elucidate the natural habitat, source, reservoir, and epidemiology of this emerging pathogen of public health significance.

\section{Acknowledgements}

We thank Prof. Dr. R.K. Narayan for going through the manuscript and Anubha for her computer help.

\section{Conflict of Interest}

None.

\section{Financial Grant}

Nil.

\section{Bibliography}

1. Rocourt J., et al. "The present state of foodborne disease in OECD countries”. Food Safety Department (2003).

2. CDC. "Food Safety". Centre for Disease Control. Atlanta, Georgia, USA (2019).

3. Pal M. "Growing significance of Vibrio parahaemolyticus as an emerging foodborne pathogen". Journal of Food Microbiology 2 (2019): 15-17.

4. Mohammed MA., et al. "Prevalence, identification and molecular characterization of Cronobacter sakazakii isolated from retail meat products". Food Control 53 (2015): 206-211.

5. Chen W., et al. "Diversity of Cronobacter spp. isolates from the vegetables in the middle-east coastline of China". World Journal of Microbiology and Biotechnology 32 (2016): 90-104.

6. Garbowska M., et al. "Microbiological quality of selected spices and herbs including the presence of Cronobacter spp". Food Microbiology 49 (2015): 1-5.

7. FAO. "Enterobacter sakazakii (Cronobacter spp.) in powdered follow-up formulae". Microbiological Risk Assessment Series 15 (2008).

8. Zimmermann J., et al. "Development of a rapid detection system for opportunistic pathogenic Cronobacter spp. in powdered milk products". Food Microbiology 42 (2014): 19-25. 
9. Xu X. "Prevalence, molecular characterization, and antibiotic susceptibility of Cronobacter spp. in Chinese ready-to-eat foods". International Journal of Food Microbiology 204 (2015): 17-23.

10. Bowen $\mathrm{AB}$ and Braden $\mathrm{CR}$. "Invasive Enterobacter sakazakii disease in infants". Emerging Infectious Diseases 12 (2006): 1185-1189.

11. Ray P., et al. "Enterobacter sakazakii in infants: Novel phenomenon in India”. Indian Journal of Medical Microbiology 25 (2007): 408-410.

12. Burdette JH and Santos C. "Enterobacter sakazakii brain abscess in the neonate: the important of neuroradiologic imaging". Pediatric Radiology 30 (2000): 33-34.

13. Lai KK. "Enterobacter infections among neonates, infants, children, and adults: case reports and a review of the literature". Medicine (Baltimore) 80 (2001):113-122.

14. Iversen $\mathrm{C}$ and Forsythe SJ. "Isolation of Enterobacter sakazakii and other Enterobacteriaceae from powdered infant formula milk and related products". Food Microbiology 21 (2004): 771777.

15. Farber JM and Forsythe SJ. "Enterobacter sakazakii". ASM Press Inc., Washington, U.S.A (2008).

16. Kandhai MC., et al. "Occurrence of Enterobacter sakazakii in food production environments and households". The Lancet 363 (2004): 39-40.

17. Mahindroo J., et al. "Cronobacter sakazakii- An unrecognized foodborne pathogen, India". International Journal of Infectious Diseases 45 (2016): 182.

18. Van Acher J., et al. "Outbreak of necrotizing enterocolitis associated with Enterobacter sakazakii in powdered milk formula". Journal of Clinical Microbiology 39 (2001): 293-297.

19. Lehner A. "Highlighting Environmental Reservoir Aspects for Cronobacter spp". Oral Presentation at the 1st International Meeting on Cronobacter (Enterobactersakazakii). University College, Dublin, Ireland (2009): 22-23.

20. Beuchat LR., et al. "Cronobacter sakazakii in foods and factors affecting its survival, growth, and inactivation". International Journal of Food Microbiology 136 (2009): 204-213.
21. Gurtler JB., et al. "Enterobacter sakazakii: a coliform of increased concern to infant health". International Journal of Food Microbiology 104 (2005): 1-34.

22. Townsend SM., et al. "Enterobacter sakazakii invades brain capillary endothelial cells, persists in human macrophages influencing cytokine secretion and induces severe brain pathology in the neonatal rat". Microbiology 153 (2007): 3538-3547.

23. Lai KK. "Enterobacter sakazakii infections among neonates, infants, children, and adults: case reports and a review of the literature". Medicine (Baltimore) 80 (2001):113-122.

24. ICMSF. "International Commission on Microbiological Specifications for Foods. Microorganisms in foods 7". Microbiological Testing in Food Safety Management (2002).

25. Muytjens HL., et al. "Quality of powdered substitutes for breast milk with regard to members of the family Enterobacteriaceae". Journal of Clinical Microbiology 26 (1988): 743-746.

26. Jung MK and Park JH. "Prevalence and thermal stability of Enterobacter sakazakii from unprocessed ready-to-eat agricultural products and powdered infant formulas". Food Science and Biotechnology 15 (2006): 152-157.

27. Agostoni C., et al. "Preparation and handling of powdered infant formula: A commentary by the ESPGHAN committee on nutrition". Journal of Pediatric, Gastroenterology and Nutrition 39 (2004): 320-322.

28. Chap J., et al. "International survey of Cronobacter sakazakii and other Cronobacter spp. in follow up formulas and infant foods". International Journal of Food Microbiology 31 (2009): 185-186.

29. Friedemann M. "Enterobacter sakazakii in food and beverages (other than infant formula and milk powder)". International Journal of Food Microbiology 116 (2007): 1-10.

30. Mullane N., et al. "Dissemination of Cronobacter spp. (Enterobacter sakazakii) in a powdered milk protein manufacturing facility". Applied and Environmental Microbiology 74 (2008): 5913-5917.

31. Fakruddin Md., et al. "Cronobacter sakazakii (Enterobacter sakazakii)". An Emerging Food borne Pathogen”. International Journal of Biomedical and Advance Research 4 (2013): 349359. 
32. Block C., et al. "Cluster of neonatal infections in Jerusalem due to unusual biochemical variant of Enterobacter sakazakii". European Journal of Clinical Microbiology and Infectious Diseases 21 (2002): 613-616.

33. Muytjens HL and Kollee LAA. "Enterobacter sakazakii meningitis in neonates: causative role in formula". The Pediatric Infectious Disease Journal 9 (1990): 372-373.

34. Yan QQ., et al. "Cronobacter species (formerly known as Enterobacter sakazakii) in powdered infant formula: a review of our current understanding of the biology of this bacterium". Journal of Applied Microbiology 11 (2012): 1-15.

Volume 2 Issue 11 November 2019

(C) All rights are reserved by Mahendra Pal., et al. 